\title{
Hippocampal Ripple-Contingent Training Accelerates Trace Eyeblink Conditioning and Retards Extinction in Rabbits
}

\author{
Miriam S. Nokia, ${ }^{1,2}$ Markku Penttonen, ${ }^{1}$ and Jan Wikgren ${ }^{1}$ \\ ${ }^{1}$ Department of Psychology, University of Jyväskylä, FI-40014 Jyväskylä, Finland, and ²Department of Psychology, Center for Collaborative Neuroscience, \\ Rutgers University, Piscataway, New Jersey 08854
}

\begin{abstract}
There are at least two distinct oscillatory states of the hippocampus that are related to distinct behavioral patterns. Theta (4-12 Hz) oscillation has been suggested to indicate selective attention during which the animal concentrates on some features of the environment while suppressing reactivity to others. In contrast, sharp-wave ripples $(\sim 200 \mathrm{~Hz})$ can be seen in a state in which the hippocampus is at its most responsive to any kind of afferent stimulation. In addition, external stimulation tends to evoke and reset theta oscillation, the phase of which has been shown to modulate synaptic plasticity in the hippocampus. Theoretically, training on a hippocampus-dependent learning task contingent upon ripples could enhance learning rate due to elevated responsiveness and enhanced phase locking of the theta oscillation. We used a brain-computer interface to detect hippocampal ripples in rabbits to deliver trace eyeblink conditioning and extinction trials selectively contingent upon them. A yoked control group was trained regardless of their ongoing neural state. Ripplecontingent training expedited acquisition of the conditioned response early in training and evoked stronger theta-band phase locking to the conditioned stimulus. Surprisingly, ripple-contingent training also resulted in slower extinction in well trained animals. We suggest that the ongoing oscillatory activity in the hippocampus determines the extent to which a stimulus can induce a phase reset of the theta oscillation, which in turn is the determining factor of learning rate in trace eyeblink conditioning.
\end{abstract}

\section{Introduction}

The hippocampal electrophysiological oscillatory state ranges from one characterized by high-frequency ripples $(\sim 200 \mathrm{~Hz})$ riding on large-amplitude sharp waves (SPWs) (Buzsáki, 1986; Ylinen et al., 1995; Chrobak and Buzsáki, 1996) to another characterized by highly regular slow theta oscillations $(4-12 \mathrm{~Hz}$ ) (for review, see Buzsáki, 2002). These states are related to distinct behavioral patterns: When hippocampal local-field potentials are dominated by theta oscillation, the animal is mostly engaged in actively acquiring information about its surroundings in a species-specific way. Hippocampal SPW ripples in turn are more likely to occur during sleep or eating, grooming, and other behaviors targeted toward the animal itself.

Both of these oscillations have been implicated in learning and memory but, supposedly, serve different aspects of the process. According to many currently influential theories about the link between neural oscillations and learning, theta oscillation is thought to reflect selective attention during which the animal obtains new information (Green and Arduini, 1954; Vanderwolf, 1969; Vinogradova et al., 1993; Buzsáki, 2002) while ripples might represent the consolidation of recently processed information (Buzsáki, 1986; Chrobak and Buzsáki, 1996). Experimental evidence suggests that both ripples and theta oscillation have a

\footnotetext{
Received April 27, 2010; revised June 24, 2010; accepted July 19, 2010.

This work was funded by grants from the Academy of Finland for J.W. (114258 and 130013) and Emil Aaltonen Foundation for M.S.N. We thank Lauri Viljanto for technical help, Michael Freeman for checking the language, and John Pearce for his comments on the early version of the manuscript.

Correspondence should be addressed to Miriam S. Nokia at the above addresses. E-mail: miriam.nokia@jyu.fi. DOI:10.1523/JNEUROSCI.2165-10.2010

Copyright $\odot 2010$ the authors $\quad 0270-6474 / 10 / 3011486-07 \$ 15.00 / 0$
}

causal relationship with learning. For instance, suppressing ripple activity by hippocampal brain stimulation during a critical period after training on a spatial learning task severely retards learning (Girardeau et al., 2009). Furthermore, administering training trials in the explicit presence or absence of hippocampal theta oscillation can either accelerate or retard, respectively, learning rate in hippocampus-dependent trace eyeblink conditioning (Griffin et al., 2004; Asaka et al., 2005).

Interestingly, there is a severalfold difference in hippocampal responsiveness between the theta and the SPW-ripple state, the hippocampus responding maximally when stimulated during the SPW-ripple state (Buzsáki, 1986). Given that the effect the training stimuli exert on the brain is one of the most basic determinants of learning rate, this leads to the question of whether learning can be expedited with ripple-contingent training or whether this effect is unique to the theta state (Griffin et al., 2004; Asaka et al., 2005). We presented one group of animals with trace eyeblink conditioning (Gormezano et al., 1962) and extinction trials occurring $\sim 200 \mathrm{~ms}$ after detection of a hippocampal ripple. Simultaneously trained animals in the yoked control (YC) group received trials regardless of their hippocampal oscillatory state. We hypothesized that the ripple-contingent $(\mathrm{R}+)$ group would learn faster in the early, hippocampus-dependent phase of conditioning (Prokasy, 1984; Kim et al., 1995) due to the elevated responsiveness to the conditioned stimulus (Buzsáki, 1986). Alternatively, as ripples occur only in the explicit absence of theta oscillation (Buzsáki, 2002) and because trace conditioning in the explicit absence of theta oscillation has been shown to retard learning (Griffin et al., 2004), the R+ group could also learn at a slower rate, implying that the theta state is selectively beneficial for learning. 


\section{Materials and Methods}

Subjects

The subjects were 28 adult male New Zealand White rabbits (Harlan Netherlands/HB Lidköpings Kaninfarm) aged $\sim 4$ months and weighing $\sim 2.9 \mathrm{~kg}$ at the time of surgery. The rabbits were housed in individual metal cages on the premises of the animal research unit of the University of Jyväskylä. Food and water were available ad libitum, and room temperature and humidity were controlled. The rabbits were maintained on a 12/12 h light/dark cycle, with lights on at 6.00 A.M. All procedures were conducted during the light portion of the cycle. All the experimental procedures were implemented in accordance with the European Communities Council Directive (86/609/EEC) on the care and use of animals for research purposes.

\section{Surgery}

Subcutaneous injections of an analgesic solution $[2 \mathrm{ml} ; 0.1 \mathrm{ml}$ of buprenorphine $0.3 \mathrm{mg} / \mathrm{ml}$ (Temgesic, Schering-Plough Europe), diluted in $1 \mathrm{ml}$ of $0.9 \% \mathrm{NaCl}]$ and of an anti-inflammatory drug $[0.1 \mathrm{ml} / \mathrm{kg}$; carprofen $50 \mathrm{mg} / \mathrm{ml}$ (Rimadyl Vet, Pfizer Animal Health)] were given $30 \mathrm{~min}$ preceding the onset of surgery. The rabbits were anesthetized with an i.m. injection of ketamine-xylazine cocktail [Ketaminol Vet, (Intervet International), $50 \mathrm{mg} / \mathrm{ml}, 7.8 \mathrm{ml}$; Narcoxyl Vet (Intervet International), 20 $\mathrm{mg} / \mathrm{ml}, 2.8 \mathrm{ml}$. An injection of $0.8 \mathrm{ml} / \mathrm{kg}$ of the cocktail was given before surgery and additional $0.8 \mathrm{ml}$ doses of either the cocktail or ketamine approximately every $20 \mathrm{~min}$ subcutaneously. Eyedrops (Oftan, Santen Oy) were used to prevent the eyes from drying. At the beginning of surgery, the rabbit was placed in a stereotaxic instrument (Kopf Instruments) with the bregma $1.5 \mathrm{~mm}$ higher than the lambda. A longitudinal incision was made to the scalp and four stainless-steel anchoring screws ( $5 \mathrm{~mm}$ anterior and $5 \mathrm{~mm}$ lateral to the bregma; $13 / 10 \mathrm{~mm}$ posterior and $5 \mathrm{~mm}$ lateral to the bregma) were attached to the skull. Two of the screws were connected together, and these served as a reference measuring point for the electrophysiological recordings.

Three monopolar recording electrodes made of Teflon-insulated stainless steel wire (bare diameter $125 \mu \mathrm{m}$ ) mounted inside 27 -gauge hypodermic stainless steel tubing were chronically implanted into the right hippocampus (for details, see Korhonen, 1991) $5 \mathrm{~mm}$ posterior and 4, 5, and $6 \mathrm{~mm}$ lateral to the bregma. During implantation, LFPs and multiunit activity were monitored to define the preferred depth of the electrode (bregma-6.5-7.2 mm). The exact depth of the electrode was based on both predetermined calculations based on a brain atlas (Bures et al., 1967) as well as changes in activity seen (LFPs) and heard (multiunit activity) during implantation. Reaching the CA1 was in most cases marked by an audible increase in cell firing. Finally, the electrodes were attached to a pin connector and the whole construction cemented in place with dental acrylic.

Analgesic (buprenorphine, see above for details) was administered every $8 \mathrm{~h}$ for the next $24-48 \mathrm{~h}$ depending on the recovery rate of the animal. A $0.1 \mathrm{ml} / \mathrm{kg}$ subcutaneous injection of metoclopramide [ $5 \mathrm{mg} /$ $\mathrm{ml}$; Primperan (Sanofi Winthrop Industrie)] was given $8 \mathrm{~h}$ after surgery to facilitate normal feeding and drinking. At least 1 week was allowed for postsurgical recovery.

\section{Conditioning procedure}

Before the experiments, the rabbits were placed in a Plexiglas restraining box located in a ventilated, electrically insulated, and sound-attenuated conditioning chamber for $\sim 20 \mathrm{~min}$ to familiarize them with the experimental situation, and to ensure the functioning of the implanted electrodes. Thereafter, experimental sessions were conducted once per day on consecutive days.

The conditioned stimulus (CS) was a $2 \mathrm{kHz}, 85 \mathrm{~dB}, 200 \mathrm{~ms}$ tone, and the unconditioned stimulus (US) was a $100 \mathrm{~ms}$ corneal airpuff with 0.35 bar source pressure and having a sound pressure level of $64 \mathrm{~dB}$. The US was delivered through a nozzle with an inner diameter of $2 \mathrm{~mm}$ placed $\sim 1 \mathrm{~cm}$ away from the eye. A fan located inside the conditioning chamber behind the rabbit created a steady background noise of $\sim 65 \mathrm{~dB}$. E-Prime (Psychology Software Tools) and LabView (National Instruments) was used to control the presentation of stimuli.

The rabbits were randomly assigned to two groups: One group received trials contingent upon hippocampal ripples $(\mathrm{R}+, n=14)$, and the

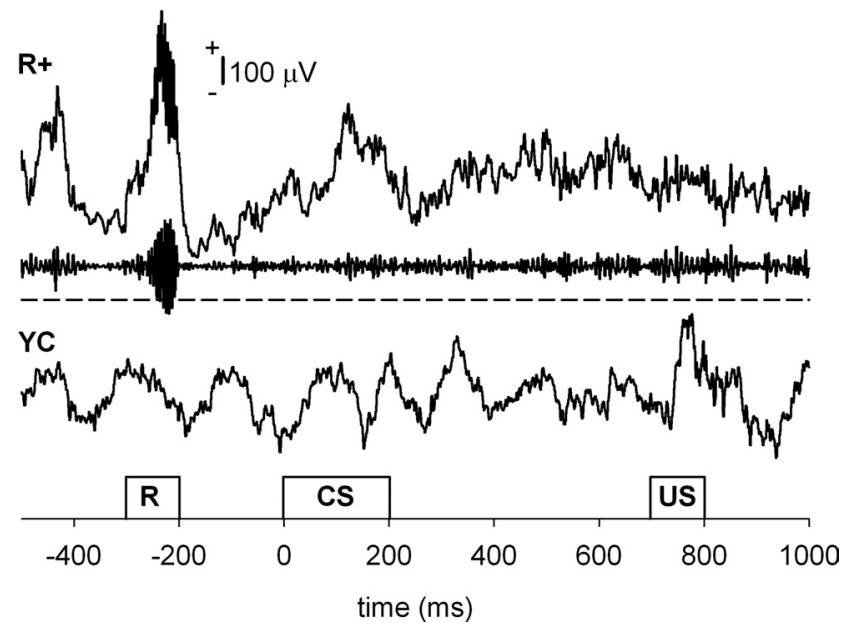

Figure 1. Trial presentation. Animals were trained in pairs, where one animal belonged to the $\mathrm{R}+$ and the other to the $\mathrm{YC}$ group. The occurrence of a ripple event was detected from the hippocampal LFP of the subject assigned to the $R+$ group by means of a simple brain-computer interface. A trial was presented simultaneously to both animals contingent upon the occurrence of a ripple in the $\mathrm{R}+$ animal. Thus, subjects in both groups received identical training. The minimum intertrial interval was set to $15 \mathrm{~s}$. For the $\mathrm{R}+$ example, both the raw (top) and the bandpass-filtered $(80-250 \mathrm{~Hz}$, middle) signals are shown. In addition, a dashed line indicates the threshold set for the ripples in this animal. For the YC example (bottom), only the raw signal is shown. $R$, Ripple detection time window.

other group was trained in parallel, thus forming a YC group $(n=14)$. The animals were first trained according to a trace conditioning paradigm for 10 sessions (paired trials) and then given 8 sessions of extinction training (CS-alone trials). The length of each daily session was limited to 50 min or 60 trials. The minimum intertrial interval was set to $15 \mathrm{~s}$. During paired trials, the CS onset preceded the US onset by $700 \mathrm{~ms}$, thus creating a $500 \mathrm{~ms}$ trace period. Before starting the extinction training, the learning outcome of each rabbit was determined: to proceed to the extinction phase, the rabbit had to meet the criterion of showing a CR in 8 of 9 consecutive trials or $>50 \%$ CRs during a single session. Rabbits showing robust learning were randomized into $\mathrm{R}+$ and $\mathrm{YC}$ groups so that half the animals in the new $\mathrm{R}+$ group had been in the $\mathrm{YC}$ group during conditioning.

To detect ripples online, the signal from the hippocampal layer CA1/ CA3 was bandpass filtered $(80-250 \mathrm{~Hz})$. Next, to ensure smooth operation of the real-time triggering system, a simple amplitude threshold for the filtered signal was set. The threshold value varied between -120 and $-400 \mu \mathrm{V}$ depending on the individual signal quality, which further depended on the exact location of the electrode tip and the variation in electrode properties. The thresholding was performed in consecutive 100 ms time windows. When the amplitude of the bandpass-filtered hippocampal signal exceeded the predetermined threshold, the brain-computer interface triggered the trial after a $200 \mathrm{~ms}$ delay (Fig. 1). A more sophisticated detection method for ripples was used in off-line analysis (see below).

\section{Recordings and data analysis}

Eyeblinks were measured using stainless steel wire hooks placed around the upper and lower eyelids for the duration of the training session to record EMG (bipolar electrode). To acquire neural measures, a low-noise preamplifier (MPA8I, Multi Channel Systems MCS) was directly attached to the electrode coupler anchored with dental acrylic to the rabbit's head. A flexible, insulated cable was used to connect the animal to the amplifier (Axon Cyberamp 380, Molecular Devices). Both the neural data and the bipolar electromyogram (EMG) were recorded with AxoScope (Molecular Devices) software and digitized (Digidata 1322A, Molecular Devices) using a $2.02 \mathrm{kHz}$ sampling rate. Before digitization, the LFPs were bandpass filtered between 0.1 and $400 \mathrm{~Hz}$, and the EMG was filtered between 30 and $300 \mathrm{~Hz}$. Clampfit (Molecular Devices), MATLAB (The MathWorks), and SPSS were used for the off-line data analyses. 
Behavioral data. The EMG signal was further high-pass filtered over $100 \mathrm{~Hz}$ and Hilbert transformed. Then an envelope curve following the peaks of the signal was calculated using the real and imaginary parts of the Hilbert transformation. For each animal and session, the baseline EMG activity was defined as the mean $(M)$ of the EMG amplitude during a 500 ms pre-CS period (MEANpre). In addition, the mean of the SD of the EMG activity during the $500 \mathrm{~ms}$ pre-CS period (SDpre) was determined. Eyeblinks were defined as EMG activity exceeding a threshold of (MEANpre $+4 \times$ SDpre). Trials showing eyeblinks during the $100 \mathrm{~ms}$ period immediately preceding CS onset were rejected. Eyeblinks during the $500 \mathrm{~ms}$ trace period were counted as conditioned responses (CRs). The learning criterion of asymptotic performance was considered to be met when the subject performed a CR on 8 of 9 consecutive paired or CS-alone trials or showed $>50 \%$ CRs during a single session. Learning rate was defined as the number of conditioning trials needed to perform the fifth CR (phase 1) (Prokasy, 1984). To define the rate of extinction, a criterion of $<30 \%$ CRs during one session was set.

To further examine early associative changes, CS-mediated reflex facilitation (Harvey et al., 1985; Weisz and LoTurco, 1988; Weisz and McInerney, 1990; Wikgren and Korhonen, 2001; Wikgren et al., 2002) was assessed by comparing unconditioned response (UR) magnitudes in the very first five trials to those in the last five trials in the first session. For this, the mean response amplitude during the $300 \mathrm{~ms}$ period following the US onset was determined. Trials showing a CR were excluded from this analysis.

Ripples. The occurrence of ripples during the 5 min presession and postsession recordings was studied off-line. For this purpose, the hippocampal LFP signal was first bandpass filtered between 80 and $250 \mathrm{~Hz}$. Next a negative threshold of $M-7 \times \mathrm{SD}$ was set for each 5 min period of each animal. The number of ripples per minute and then the change (percentage) in the number of ripples per minute from presession to postsession recordings was calculated (Eschenko et al., 2008).

Theta-band phase locking. To assess the accuracy in time of the thetaband response to the conditioning stimuli, a phase-locking value (PLV) (see Palva et al., 2005) was calculated. The PLV is based on amplitudenormalized phase information. Thus, the PLV is relatively resistant to large-amplitude events and better reflects small amplitude but well timelocked events. The PLV also allows one to obtain more comparable data from multiple subjects/recording points since it is only dependent on the consistency of the signal within one subject/recording point. The hippocampal LFP data were first bandpass filtered between 4 and $8 \mathrm{~Hz}$. A wider filter $(4-12 \mathrm{~Hz})$ was also tested for this purpose, but it did not make any difference on the results of the analysis. Then a Hilbert transform was run on the signal to obtain the phase information, and next the amplitude of the signal was normalized to 1 by dividing each data point by its absolute value. Finally, the PLV was obtained by averaging over 60 trials ( 1 session) and taking the absolute value of the mean. The PLV varies between 0 and 1, 0 indicating no phase locking and 1 indicating perfect phase locking. A simulated data of 60 random samples $(\times 100,000)$ of noise generated by a computer (LabView software) was used to estimate the 99th percentile of the PLV distribution to evaluate the statistical significance of the real PLVs. In the simulated dataset, the 99th percentile was set at PLV $=0.28$. The mean value of the PLV during the presentation of the CS (200 ms, CS-evoked PLV) was used in comparing the accuracy of the theta-band responses between groups and across learning.

Statistical analyses. For the purpose of statistical testing, behavioral data from the 10 conditioning and 8 extinction sessions were com-
B

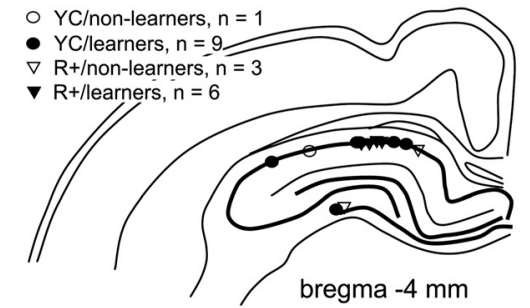

bregma $-5 \mathrm{~mm}$

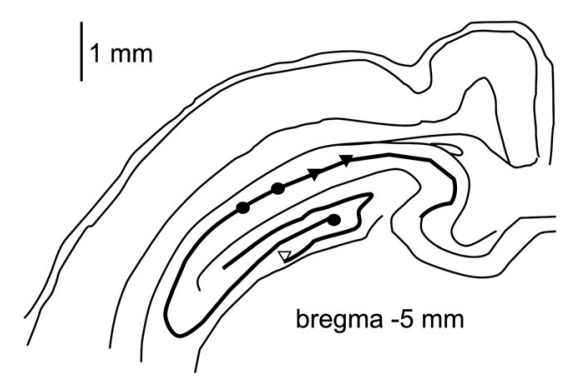

bregma $-4 \mathrm{~mm}$

ts had at least one recording electrode correctly placed in the right dorsal hippocampus and showing ripple activity. $A$, Electrode locations by group $(\mathrm{YC}$ and $\mathrm{R}+$ ) and learning during conditioning. $A$ CR on 8 of 9 consecutive paired trials or $>50 \%$ CRs during at least one conditioning session was used as the criterion for learning. $B$, Electrode locations by group and learning during extinction. Less than $30 \%$ CRs during at least one training session was used as

pressed into blocks of 2 sessions. The neural data were divided according to the learning phase. A learning criterion of fifth CR for conditioning and a criterion of $<30 \%$ CRs during one training session for extinction were used as division points. Data from the sessions before meeting the criterion were averaged (before), as were data from the exact session during which the criterion was met (during) and from the sessions following the fulfillment of the criterion (after). Repeated-measures ANOVA with block/phase as a within-subjects factor, and group as a between-subject factor, was used in analyzing changes across the training. Greenhouse-Geisser corrected degrees of freedom were used whenever the sphericity assumption was violated. One-way ANOVA was used in comparing the groups block by block/phase by phase. One-sample $t$ test was used to evaluate the significance of the change in ripple rate per minute between the presession and postsession recordings.

\section{Histology}

After the experiments, the rabbits were anesthetized with an intramuscular injection of ketamine-xylazine cocktail and then overdosed with an intravenous injection of pentobarbital (Mebunat Vet, Orion-Yhtymä Oyj). Next, the brain was perfused by putting physiological saline followed by $10 \%$ formalin through the ascending aorta. The locations of the electrode tips were marked by passing a DC current $(200 \mu \mathrm{A}, 20 \mathrm{~s})$ through the electrode. The brain was then removed and stored in $10 \%$ formalin $+10 \%$ sucrose solution for $\sim 1$ week. Next, the brain was frozen and coronally sectioned with a microtome into $100-\mu \mathrm{m}$-thick slices. The slices were attached to gelatinized slides and later stained with Prussian blue and cresyl violet. The electrode-tip locations were determined from the stained slides with the help of a microscope and a stereotaxic atlas (Bures et al., 1967).

\section{Results}

\section{Histology}

All 28 subjects had at least one recording electrode in the right dorsal hippocampus showing ripple activity (Fig. 2).

\section{Ripple-contingent training resulted in faster conditioning and slower extinction}

The average ITI was $40.89 \mathrm{~s}(\mathrm{SEM}=0.44 \mathrm{~s}, \min =17.30 \mathrm{~s}$, and $\max =267.02 \mathrm{~s})$ during conditioning and $39.10 \mathrm{~s}(\mathrm{SEM}=0.40 \mathrm{~s}$, $\min =17.00 \mathrm{~s}$, and $\max =221.95 \mathrm{~s}$ ) during extinction training. 
A

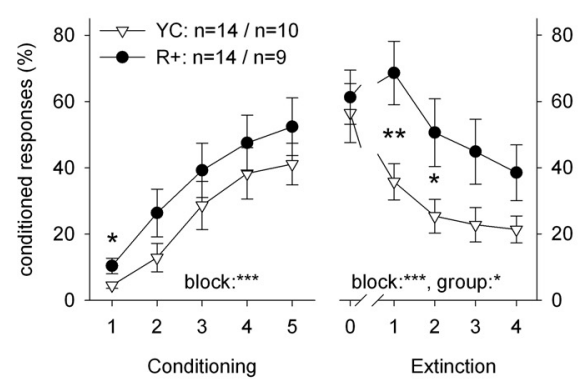

C

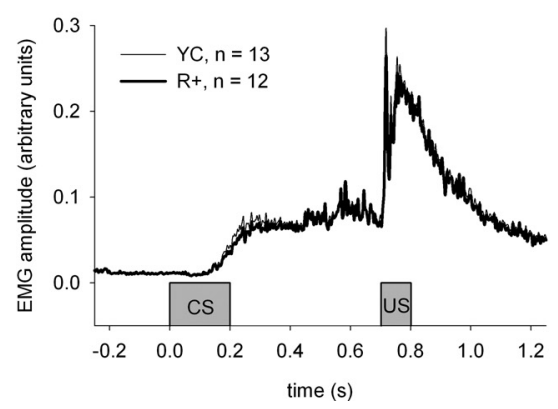

B

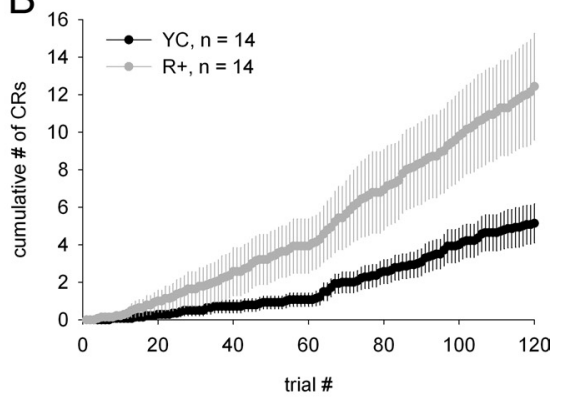

D

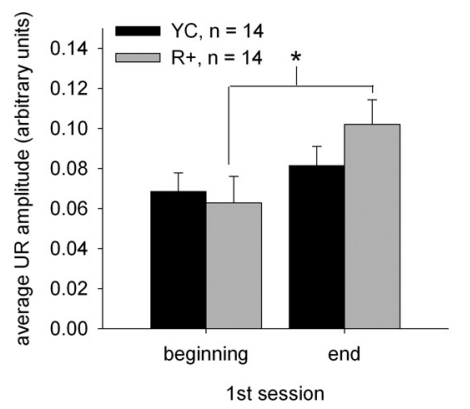

Figure 3. Ripple-contingent training led to faster learning and slower extinction. $\boldsymbol{A}$, CRs as a function of training block. Each block represents the average $(R$ percentage during two consecutive daily 60 -trial sessions. During conditioning, rabbits in the $R+$ group ( $n=14$ ) received paired presentations of the CS (tone) and the US (airpuff) contingent upon hippocampal ripples, while rabbits in the YC $(n=14)$ group received conditioning trials regardless of their neural state. A 500 ms trace period separated the $C S$ and the US during each conditioning trial. Rabbits in both groups acquired a robust $C R$, although rabbits in the $R+$ group showed more CRs during the very early phase of conditioning (see left panel, first block). Also, more animals in the $R+$ group ( 12 of 14) reached asymptotic learning than in the YC group (7 of 14). Extinction training was administered only in rabbits showing robust learning at the end of conditioning $(n=19)$. These rabbits that learned well from the original $\mathrm{R}+$ and $Y C$ groups were divided into $\mathrm{R}+(n=9)$ and $Y C(n=10)$ groups in a counterbalancing manner. During extinction training, the tone-CS was always presented alone. Extinction training reduced the number of CRs in both groups, but more so in the YC group. That is, extinction was slower in rabbits in the $R+$ group. Block 0 in the right panel represents $C R$ percentage during the last block of conditioning training. $\boldsymbol{B}$, The cumulative number of $C R$ s plotted as a function of trial number in the $R+$ and $Y$ C groups separately shows that there is no initial difference in responding. Vertical lines depict the SEM. $\boldsymbol{C}$, The average response topographies of the two groups during the first two sessions (block 1) were notably similar. Both groups show correctly timed CRs that peak around US onset. Only trials during which an animal emitted a CR were included in these averages. $\boldsymbol{D}$, Reflex facilitation was stronger in the $\mathrm{R}+$ group. Asterisks refer to statistical significance: ${ }^{*} p<0.05,{ }^{* *} p<0.01,{ }^{* * *} p<0.001$.

Overall, conditioning led to the acquisition of a CR in a similar manner in the $\mathrm{R}+(n=14)$ and $\mathrm{YC}(n=14)$ groups, as shown by the results of a repeated-measures ANOVA: block: $F_{(4,104)}=$ 24.46, $p<0.001$; group: ns; block $\times$ group: ns (Fig. $3 A, C$ ). However, as expected, rabbits in the $\mathrm{R}+$ group showed more CRs during the early phase of conditioning (block $1: F_{(1,26)}=5.40$, $p<0.05$ ) (Fig. $3 A, B$ ). The mean number of trials to the fifth CR was $119(\mathrm{SEM}= \pm 13.12$, $\min =13$, and $\max =297)$. Rabbits in the $\mathrm{R}+$ group acquired the fifth $\mathrm{CR}$ in $94 \pm 14.51$ trials and those in the YC group in $144 \pm 20.24$ trials $\left(F_{(1,26)}=3.97, p=0.057\right)$. Nineteen of the twenty-eight animals acquired a robust CR during the 10 sessions of trace eyeblink conditioning. Seven of the nine animals that did not learn were in the YC group, indicating that more animals in the $\mathrm{R}+$ group reached asymptotic learning (12 of 14).

Unconditioned responses emitted during the first conditioning session were of greater magnitude in the last five trials than in the first five trials $\left(F_{(1,26)}=7.44, p<0.05\right)$, indicating CS-mediated reflex facilitation. However, paired samples $t$ test revealed a significant increase in UR magnitude only in the $\mathrm{R}+$ group: $t_{(13)}=2.26, p<$ 0.05 (Fig. 3D).

Animals that did not meet the learning criterion were excluded from the extinction phase. The ones that did learn were

regrouped into $\mathrm{R}+(n=9)$ and $\mathrm{YC}(n=$ 10) groups for extinction so that belonging to either group during preceding conditioning was counterbalanced. Extinction training reduced the number of CRs in both groups, but more so in the YC group: block: $F_{(4,68)}=15.03, p<0.001$; group: $F_{(1,17)}=4.96, p<0.05$; block $\times$ group: ns (Fig. 3). Rabbits in the $\mathrm{R}+$ group showed more CRs than rabbits in the YC group, especially early during extinction training (block 1: $F_{(1,17)}=9.32$, $p<0.01$; block 2: $\left.F_{(1,17)}=5.20, p<0.05\right)$, that is, extinction was slower when trials were administered contingent upon hippocampal ripples.

\section{The rate of hippocampal ripples per minute increased in response to the training session in the yoked control group early in conditioning, before learning}

The rate of ripples per minute varied between 0 and 45.21, with an average of 9.09 ripples/min $(\mathrm{SEM}= \pm 0.17)$. Although the rabbits were randomly assigned to the two groups, the $\mathrm{R}+$ group consistently showed more ripples ( $M=10.76$ ripples/ $\min , \mathrm{SEM}= \pm 0.33)$ than the YC group $(M=8.15$ ripples $/ \mathrm{min}, \mathrm{SEM}= \pm 0.28)$ during conditioning. The same was true during extinction training: 7.69 ripples/ $\min (\mathrm{SEM}= \pm 0.36)$ in the YC group and $9.43 \mathrm{ripples} / \mathrm{min}(\mathrm{SEM}= \pm 0.36)$ in the $\mathrm{R}+$ group. However, since the change (percentage) between the presession and postsession recordings was used in the analyses, this did not pose a problem.

During conditioning, in two animals in the YC group very few ripples were detected ( $<2$ per minute), and these animals were thus excluded from the further analyses. During extinction, very few ripples $(<2$ per minute) could be identified in one animal in the $\mathrm{YC}$ group, and it was thus discarded. Also, animals not showing robust CRs at the end of conditioning or not showing extinction (CR rate did not fall below 30\%) were excluded from the further analyses on hippocampal ripples. Thus, for analyses on ripples the number of subjects in the $\mathrm{R}+$ group was 14 for conditioning and 5 for extinction. The number of subjects in the YC groups was 12 and 8, respectively. Using repeated-measures ANOVAs, no changes in the presession versus postsession change across learning were detected in either group during either conditioning or extinction (Fig. 4A). However, rabbits in the YC group showed an increase in ripple rate per minute in response to the training session (presession vs postsession) early in conditioning, before they reached the fifth CR $\left(t_{(11)}=2.48, p<0.05\right)$.

\section{Hippocampal theta-band activity showed stronger phase locking to the CS in the ripple-contingent group early in conditioning, before learning}

Only subjects with electrodes in the CA1 region $(n=24)$ were included in the analyses regarding theta activity. Animals not showing robust CRs at the end of conditioning or not showing 
extinction (CR rate did not fall below $30 \%$ ) were excluded from the extinction training analyses. Thus, the number of subjects in the $\mathrm{R}+$ group included in analyses was 13 for conditioning and 5 for extinction. The number of subjects in the YC groups was 11 and 7, respectively. Hippocampal theta-band activity showed consistent and statistically significant phase locking to the conditioning stimuli, as indicated by the PLVs shown in Figure $4, B$ and $C$. In the simulated data, the 99th percentile of the PLV was set at 0.28 . This means that with an $\alpha$ of 0.01 , the PLV is statistically significant when it is $>0.28$. The CS-related PLV (Fig. 4B) showed no changes across learning either during conditioning or extinction, but the PLVs were higher in the $\mathrm{R}+$ group than in the $\mathrm{YC}$ group $\left(F_{(1,22)}=5.11, p<0.05\right)$. This group difference was greatest before learning $\left(F_{(1,22)}=9.73, p<0.01\right)$. It is notable that the PLV might also reflect evoked potentials (EPs) but as seen in Figure 4, the width of the PLV peak clearly surpasses duration of an EP, and the PLV in the $\mathrm{R}+$ group also remains significant for the duration of ISI, which is not the case in the YC group.

\section{Discussion}

We showed that presenting training trials systematically following a hippocampal SPW ripple led to faster acquisition of the $\mathrm{CR}$ in the initial phase of learning in trace eyeblink conditioning in rabbits. In addition, we saw stronger CS-evoked phase locking of hippocampal theta oscillation in the ripple-contingent group than in the group receiving training trials regardless of the ongoing neural state. This effect was only significant early in learning, concomitant with the onset of $\mathrm{CR}$ acquisition. Ripple-contingent training also retarded extinction rate when the CS was presented alone to well trained animals. Last, conditioning increased the rate of ripples but only in the yoked control group early in learning.

These results imply that the neural state characterized by the dominance of hippocampal theta oscillation is not a uniquely beneficial state for learning (Seager et al., 2002; Griffin et al., 2004; Asaka et al., 2005). This is especially noteworthy since SPW ripples only occur in the explicit absence of theta oscillation (Buzsáki, 2002), and the two oscillatory states are thought to indicate different modes of information processing (Buzsáki, 1989). Thus, although probably not the crucial factor in determining learning rate, the relative magnitude of the spontaneous theta oscillation could nevertheless be indicative of a somewhat permanent disposition of an individual and thus indirectly affect learning rate by regulating the responsiveness of the animal.

In addition to a faster learning rate, we found stronger phase locking (Palva et al., 2005) of hippocampal theta oscillations in response to the CS early in training in the ripple-contingent group. Previously, CS-evoked theta-band phase locking (Siegel et al., 2009) and theta oscillation in general (Nokia et al., 2009) have been shown to strengthen immediately preceding the onset of $\mathrm{CR}$
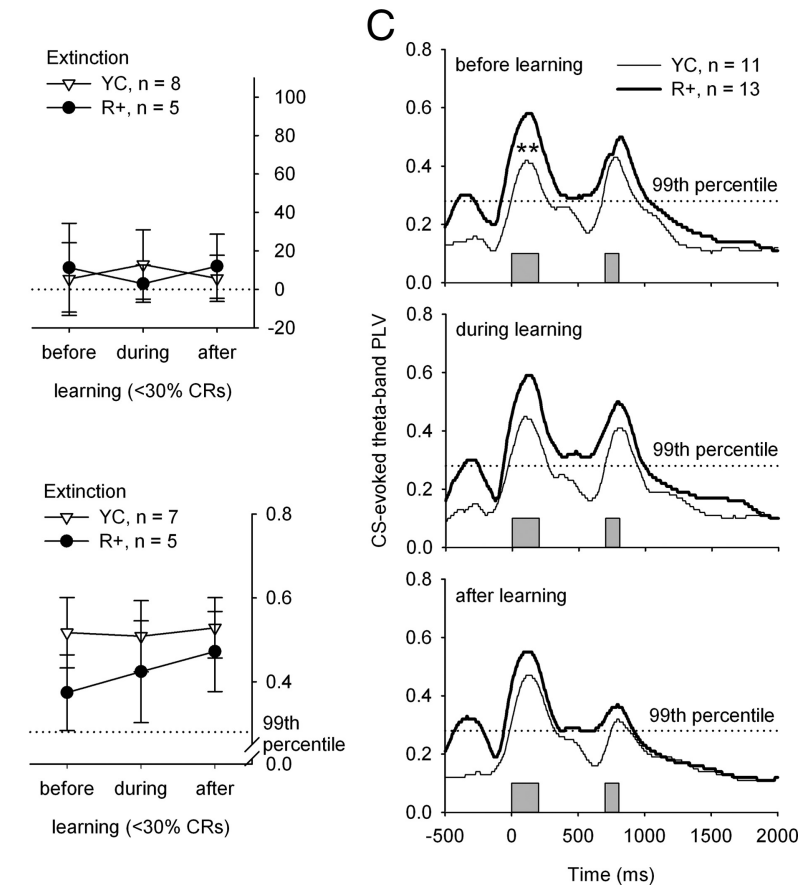

Figure 4. $\quad \boldsymbol{A}, \boldsymbol{B}$, Change (percentage) in hippocampal ripple rate $(\boldsymbol{A})$ and theta-band phase locking $(\boldsymbol{B})$ across learning. The data were divided into three sections (before, during, and after learning) based on the session during which the fifth $C R$ was seen (conditioning) or when the animal showed $<30 \%$ conditioned responses (extinction). $\boldsymbol{A}$, The presession versus postsession (percentage) in ripple rate per minute showed no differences between the $Y($ and the $R+$ group, nor did it show an The theta-band PLV across time during each phase of learning during conditioning (compare to $\boldsymbol{B}$, left panel). Note that the width 列 duration of ISI, which is not the case in the YC group. The gray bars at the bottom indicate the timing of the CS and the unconditioned stimulus. The peak in the PLVs of the $\mathrm{R}+$ group occurring immediately before the $\mathrm{CS}$ reflects the sharp-wave component of the SPW-ripple complex. Asterisks refer to statistical significance: ${ }^{*} p<0.05,{ }^{* *} p<0.01$.

acquisition in the course of trace eyeblink conditioning. At the neuronal level, phase locking of neural oscillations within a structure, as well as synchronized oscillations between functionally related brain structures, allows predictability in terms of neuronal communication. This predictability probably plays a key role in the formation of transient neural assemblies relevant for a particular task (Fries, 2005; Sauseng and Klimesch, 2008). Interestingly, it was recently shown that theta oscillations exist also in the cerebellum (Hoffmann and Berry, 2009; Wikgren et al., 2010), the brain structure critical in all eyeblink conditioning (Thompson and Steinmetz, 2009), and that these oscillations are synchronized with those seen in the hippocampus, in particular immediately after the presentation of external stimuli. Further, theta-band phase locking/reset has also been suggested to facilitate the optimal induction of long-term potentiation (LTP) (Givens, 1996; Williams and Givens, 2003; McCartney et al., 2004). In fact, the phase of the theta oscillation seems to regulate synaptic plasticity by either enhancing or preventing LTP (Hölscher et al., 1997). In addition to the direct link often made between LTP and the storage of memories, LTP has also been proposed to function as a neural equivalent of arousal or attention (Shors and Matzel, 1997). Further, theta-band phase locking in itself has been suggested to indicate selective attention to certain stimuli (Vinogradova et al., 1993). In sum, it is reasonable to assume that faster conditioning in the ripple-contingent group is due to enhanced CS-evoked theta-band phase locking enabled by a maximal state 
of responsiveness reflecting, at the neuronal level, augmented formation of transient functional neural assemblies and, at the psychological level, increased selective attention directed at the CS.

Acquisition of the CR in trace eyeblink conditioning requires intact hippocampal functioning; this is most likely because hippocampus is a structure crucially involved in the establishment of the CS-US contingency association (Shors, 2004), which is hypothesized to be phase 1 in the learning process (Prokasy, 1984). In fact, human studies show that trace eyeblink conditioning is impossible if the participant fails to verbally report the contingency of the CS and US (Clark and Squire, 1998) or severely retarded in the case of bilateral lesions of the medial temporal lobe (McGlinchey-Berroth et al., 1997). Other limbic structures, like the amygdala, have also been shown to modulate learning rate, possibly through rapidly emerging emotional associations (Neufeld and Mintz, 2001), which are seen, for example, as CSmediated reflex facilitation (e.g., Wikgren et al., 2002). Thus, the hippocampus is involved in rapidly emerging learning-related plasticity, which affects the rate at which the CR is acquired. In the light of our results, we might assume that the heightened responsiveness of the hippocampus in the SPW-ripple state and the greater theta-band phase locking to the CS enhance learning by virtue of synchronizing activity in task relevant areas and, perhaps, by providing the cerebellar learning circuit with adequate information relatively early in training. Indeed, it has been shown that direct pontine nuclei (the origin of the CS pathway to the cerebellum) stimulation can be used as a "supernormal" CS in classical conditioning (Steinmetz et al., 1986).

Interestingly, ripple-triggered training slowed down extinction, a process that, like trace eyeblink conditioning, is affected by hippocampal lesions (Moyer et al., 1990). This is at odds with faster acquisition during initial conditioning, since the gradual loss of CRs during extinction training is generally thought to reflect an active learning process as well. The majority of modern learning theories assert that either the CS intensity/salience (Rescorla and Wagner, 1972) or attention paid to it (Mackintosh, 1975; Pearce and Hall, 1980; Wagner, 1981) determines learning rate during conditioning, stronger stimuli leading to faster learning. This is in line with our results on conditioning discussed above but in contrast with the result regarding extinction. As far as we know, there is no direct experimental evidence on the effect of CS intensity/salience on extinction rate, nor has theta-contingent extinction training been reported. It would be interesting to see whether such a manipulation would have a differential effect in comparison to ripple-triggered extinction training.

One possible explanation for why ripple-contingent training facilitates conditioning but retards extinction is that while the neural state reflected by the occurrence of an SPW ripple promotes excitatory responding to stimulation (Buzsáki, 1986), it might at the same time impede long-term depression or other forms of responding in the hippocampus. Thus, presenting conditioning stimuli contingent upon hippocampal SPW ripples could make neural responding less flexible, favoring only those forms of learning that depend mostly on excitatory responding in the hippocampus.

An alternative explanation for faster learning but slower extinction in the ripple-contingent group would be that the SPW state enhances only performance, and not learning. However, the facts that (1) rabbits in neither group emitted responses to the CS at the very outset of conditioning but gradually developed a CR, (2) the response topographies of animals in both groups were highly similar, suggesting that the eyeblinks emitted by rabbits in the $\mathrm{R}+$ group were proper CRs and not alpha reactions, and (3) both groups showed CS-mediated reflex facilitation early in conditioning, the effect being even stronger in the $\mathrm{R}+$ group, all suggest this was not the case (Fig. 3). Thus, while the theoretical possibility remains that both groups learned equally fast but the $\mathrm{R}+$ group manifested learning more readily, we think it is justified to conclude that the $\mathrm{R}+$ group learned faster, at least in behaviorist terms.

With respect to the changes in the hippocampal ripple rate in the present experiment, we saw an increase in ripple rate in response to the training session early in learning during conditioning, but only in the yoked control group. A training-induced increase in ripple rate (Eschenko et al., 2008) has been found previously when recordings were made in rats following a spatial learning task, over a longer period of time, during sleep. These differences in methods probably account at least partially for the different results, i.e., the absence of an increase in postsession ripple rate in the ripple-contingent group obtained in our study. Another explanation might be that for subjects in the $\mathrm{R}+$ group the conditioning task was easier, as suggested by faster learning in this group. Accordingly, increased ripple activity after the learning event might not be necessary during easier tasks when learning occurs rapidly. To summarize, our findings are in line with the current theoretical idea that novel and important recent experiences are replayed in the hippocampus afterward, as indicated by the increased occurrence of ripples after training.

In conclusion, we propose that the rate of associative learning in a hippocampus-dependent task can be affected by presenting the conditioning stimuli selectively at a moment when the hippocampus is free from subcortical control, as marked by the relative absence of theta oscillations and the occurrence of an SPW ripple. It is at this moment that the hippocampus is most responsive to external stimulation, resulting in a more synchronized theta-band oscillatory response probably leading to enhanced information flow between learning-related brain structures, and on the psychological level, in increased perceived salience of and selective attention toward the stimuli, both affecting learning rate. The ongoing hippocampal oscillatory state appears to regulate or indicate the degree to which training stimuli are able to elicit a synchronized theta-band oscillation, which in turn seems to determine the learning rate early in training.

\section{References}

Asaka Y, Mauldin KN, Griffin AL, Seager MA, Shurell E, Berry SD (2005) Nonpharmacological amelioration of age-related learning deficits: the impact of hippocampal theta-triggered training. Proc Natl Acad Sci U S A 102:13284-13288.

Bures J, Petran M, Zachar J (1967) Electrophysiological methods in biological research. New York: Academic.

Buzsáki G (1986) Hippocampal sharp waves: their origin and significance. Brain Res 398:242-252.

Buzsáki G (1989) Two-stage model of memory trace formation: a role for "noisy" brain states. Neuroscience 31:551-570.

Buzsáki G (2002) Theta oscillations in the hippocampus. Neuron 33:325-340.

Chrobak JJ, Buzsáki G (1996) High-frequency oscillations in the output networks of the hippocampal-entorhinal axis of the freely behaving rat. J Neurosci 16:3056-3066.

Clark RE, Squire LR (1998) Classical conditioning and brain systems: the role of awareness. Science 280:77-81.

Eschenko O, Ramadan W, Mölle M, Born J, Sara SJ (2008) Sustained increase in hippocampal sharp-wave ripple activity during slow-wave sleep after learning. Learn Mem 15:222-228.

Fries P (2005) A mechanism for cognitive dynamics: neuronal communication through neuronal coherence. Trends Cogn Sci 9:474-480.

Girardeau G, Benchenane K, Wiener SI, Buzsáki G, Zugaro MB (2009) 
Selective suppression of hippocampal ripples impairs spatial memory. Nat Neurosci 12:1222-1223.

Givens B (1996) Stimulus-evoked resetting of the dentate theta rhythm: relation to working memory. Neuroreport 8:159-163.

Gormezano I, Schneiderman N, Deaux E, Fuentes I (1962) Nictitating membrane: classical conditioning and extinction in the albino rabbit. Science 138:33-34.

Green JD, Arduini AA (1954) Hippocampal electrical activity in arousal. J Neurophysiol 17:533-557.

Griffin AL, Asaka Y, Darling RD, Berry SD (2004) Theta-contingent trial presentation accelerates learning rate and enhances hippocampal plasticity during trace eyeblink conditioning. Behav Neurosci 118:403-411.

Harvey JA, Gormezano I, Cool-Hauser VA (1985) Relationship between heterosynaptic reflex facilitation and acquisition of the nictitating membrane response in control and scopolamine-injected rabbits. J Neurosci 5:596-602.

Hoffmann LC, Berry SD (2009) Cerebellar theta oscillations are synchronized during hippocampal theta-contingent trace conditioning. Proc Natl Acad Sci U S A 106:21371-21376.

Hölscher C, Anwyl R, Rowan MJ (1997) Stimulation on the positive phase of hippocampal theta rhythm induces long-term potentiation that can be depotentiated by stimulation on the negative phase in area CA1 in vivo. J Neurosci 17:6470-6477.

Kim JJ, Clark RE, Thompson RF (1995) Hippocampectomy impairs the memory of recently, but not remotely, acquired trace eyeblink conditioned responses. Behav Neurosci 109:195-203.

Korhonen T (1991) A method for rapid implantation of multielectrode systems. Physiol Behav 49:401-403.

Mackintosh NJ (1975) A theory of attention: variations in the associability of stimuli with reinforcement. Psychol Rev 82:276-298.

McCartney H, Johnson AD, Weil ZM, Givens B (2004) Theta reset produces optimal conditions for long-term potentiation. Hippocampus 14:684687.

McGlinchey-Berroth R, Carrillo MC, Gabrieli JD, Brawn CM, Disterhoft JF (1997) Impaired trace eyeblink conditioning in bilateral, medialtemporal lobe amnesia. Behav Neurosci 111:873-882.

Moyer JR Jr, Deyo RA, Disterhoft JF (1990) Hippocampectomy disrupts trace eye-blink conditioning in rabbits. Behav Neurosci 104:243-252.

Neufeld M, Mintz M (2001) Involvement of the amygdala in classical conditioning of eyeblink response in the rat. Brain Res 889:112-117.

Nokia MS, Penttonen M, Korhonen T, Wikgren J (2009) Hippocampal theta-band activity and trace eyeblink conditioning in rabbits. Behav Neurosci 123:631-640.

Palva JM, Palva S, Kaila K (2005) Phase synchrony among neuronal oscillations in the human cortex. J Neurosci 25:3962-3972.

Pearce JM, Hall G (1980) A model for Pavlovian learning: variations in the effectiveness of conditioned but not of unconditioned stimuli. Psychol Rev 87:532-552.

Prokasy WF (1984) Presidential address, 1983. Psychophysiology 21:1-13.

Rescorla RA, Wagner AR (1972) A theory of Pavlovian conditioning: variations in the effectiveness of reinforcement and nonreinforcement. In:
Classical conditioning II (Black AH, Prokasy WF, eds), pp 64-99. New York: Appleton-Century-Crofts.

Sauseng P, Klimesch W (2008) What does phase information of oscillatory brain activity tell us about cognitive processes? Neurosci Biobehav Rev 32:1001-1013.

Seager MA, Johnson LD, Chabot ES, Asaka Y, Berry SD (2002) Oscillatory brain states and learning: impact of hippocampal theta-contingent training. Proc Natl Acad Sci U S A 99:1616-1620.

Shors TJ (2004) memory traces for trace memories: neurogenesis, synaptogenesis and awareness. Trends Neurosci 27:250-256.

Shors TJ, Matzel LD (1997) Long-term potentiation: what's learning got to do with it? Behav Brain Sci 20:597-614; discussion 614-655.

Siegel JJ, Rosin LM, Tat C, Davis TK, Mauk MK (2009) Hippocampal theta reset precedes the acquisition of conditioned responses in trace eyelid conditioning. Soc Neurosci Abstr 35:384.7.

Steinmetz JE, Rosen DJ, Chapman PF, Lavond DG, Thompson RF (1986) Classical conditioning of the rabbit eyelid response with a mossy-fiber stimulation CS: I. Pontine nuclei and middle cerebellar peduncle stimulation. Behav Neurosci 100:878-887.

Thompson RF, Steinmetz JE (2009) The role of the cerebellum in classical conditioning of discrete behavioral responses. Neuroscience 162:732755.

Vanderwolf CH (1969) Hippocampal electrical activity and voluntary movement in the rat. Electroencephalogr Clin Neurophysiol 26:407-418.

Vinogradova OS, Brazhnik ES, Kitchigina VF, Stafekhina VS (1993) Acetylcholine, theta-rhythm and activity of hippocampal neurons in the rabbit-IV. sensory stimulation. Neuroscience 53:993-1007.

Wagner AR (1981) SOP: A model of automatic memory processing in animal behavior. In: Information processing in animals: memory mechanisms (Spear NE, Miller RR, eds), pp 5-47. Hillsdale, NJ: Erlbaum.

Weisz DJ, LoTurco JJ (1988) Reflex facilitation of the nictitating membrane response remains after cerebellar lesions. Behav Neurosci 102:203-209.

Weisz DJ, McInerney J (1990) An associative process maintains reflex facilitation of the unconditioned nictitating membrane response during the early stages of training. Behav Neurosci 104:21-27.

Wikgren J, Korhonen T (2001) Interpositus nucleus inactivation reduces unconditioned response amplitude after paired but not unpaired treatment in rabbit eyeblink conditioning. Neurosci Lett 308:181-184.

Wikgren J, Ruusuvirta T, Korhonen T (2002) Reflex facilitation during eyeblink conditioning and subsequent inactivation of the dentate-interpositus nucleus in the rabbit (Oryctolagus cuniculus). Behav Neurosci 116:1052-1058.

Wikgren J, Nokia MS, Penttonen M (2010) Hippocampo-cerebellar theta band phase synchrony in rabbits. Neuroscience 165:1538-1545.

Williams JM, Givens B (2003) Stimulation-induced reset of hippocampal theta in the freely performing rat. Hippocampus 13:109-116.

Ylinen A, Bragin A, Nádasdy Z, Jandó G, Szabó I, Sik A, Buzsáki G (1995) Sharp wave-associated high-frequency oscillation $(200 \mathrm{~Hz})$ in the intact hippocampus: network and intracellular mechanisms. J Neurosci 15: $30-46$. 